\title{
Percepção dos consumidores em relação à estratégia de PRODUCT PLACEMENT em séries de TV: Um estudo comparativo entre brasileiros e americanos
}

\section{Consumer perception about PRODUCT PLACEMENT'S strategy in TV series: A comparative study between brazilian and american}

\author{
Greici Galiotto ${ }^{1}$ \\ Fernanda Lazzari² \\ Tatiana Piccin ${ }^{3}$ \\ Luciene Eberle ${ }^{4}$
}

\begin{abstract}
Resumo
O uso do product placement em séries de TV tem crescido substancialmente nos últimos anos e, por isso, existe a necessidade de entender melhor como o público percebe o uso dessa estratégia de marketing. Frente a isso, este trabalho objetiva analisar a percepção que brasileiros e americanos têm em relação ao uso de product placement em séries de TV. Para o desenvolvimento do estudo, realizou-se uma pesquisa quantitativa com brasileiros e americanos, a qual foi aplicada, principalmente, através de redes sociais. Além de questões de perfil do consumidor, investigaram-se os seguintes construtos: percepção geral do uso de product placement em séries de TV; aceitação do seu uso; atitudes para com o seu uso; interesse e a intenção de compra que o product placement desperta. Verificou-se que brasileiros e americanos têm boa aceitação do uso da estratégia. Americanos apresentam atitudes mais favoráveis ao uso de product placement, enquanto brasileiros têm uma percepção geral mais positiva e também apresentam maior interesse na marca e maior intenção de compra despertada através do uso de placements.
\end{abstract}

Palavras-chave: Product placement. Brasileiros. Americanos. Séries de TV.

\begin{abstract}
The use of product placement in TV shows has grown substantially in the past few years, therefore, there is a necessity of understanding better how the audience feel about this marketing strategy. Therefore, this study aims to analyze brazilian's and american's perception regarding the use of product placement in TV shows. For this, a quantitative survey was applied with brazilians and americans, mostly, through social media. Besides consumer profile questions, the following constructs were investigated: general perception of use of product placement in TV shows; the acceptance of its use; atitudes toward its use; brand interest and purchase intention evoked by product placement. It was found that both brazilians and americans have good acceptance of the strategy. Americans have more favorable attitudes toward the use of product placement, while brazilians have a more positive general perception, and also have more brand interest and purchase intention, evoked by placements.
\end{abstract}

Keywords: Product placement. Brazilians. Americans. TV shows.

Graduação em andamento em Comércio Internacional na Universidade de Caxias do Sul, UCS. Brasil. Afiliação: Universidade de Caxias do Sul. Lattes: http://lattes.cnpq.br/7752013002027062 Email: greici.14@hotmail.com

2 Doutora em Administração pela Universidade Federal do Rio Grande do Sul, na área de concentração de Marketing. Mestre em Administração pela Universidade de Caxias do Sul. Bacharel em Administração pela Universidade de Caxias do Sul. Licenciada em Matemática pela Universidade de Caxias do Sul. Brasil. Afiliação: Universidade de Caxias do Sul. Lattes: http://lattes.cnpq.br/8433623789354128 Email: fernandalazzari@hotmail.com

3 Graduação em andamento em Comércio Internacional na Universidade de Caxias do Sul, UCS. Brasil. Afiliação: Universidade de Caxias do Sul Lattes: http://lattes.cnpq.br/5041861853212604 Email: piccintati@gmail.com

4 Doutora em Administração pela Associação Ampla PUCRS - Pontifícia Universidade Católica do Rio Grande do Sul e UCS - Universidade de Caxias do Sul. Brasil. Afiliação: Universidade de Caxias do Sul. Lattes: http://lattes.cnpq.br/9510755031322359 Email: Leberle@ucs.br 


\section{Introdução}

Objetivando promover uma troca eficaz de produtos e serviços entre produtor e consumidor, a CIM (Comunicação Integrada de Marketing) possibilita a evolução da comunicação massiva para uma comunicação personalizada, que inclui diversas técnicas e métodos específicos para cada área (AMARAL, 2008). Considerando que a publicidade dispõe de inúmeras ferramentas para a transmissão da mensagem da marca aos consumidores, Shimp (2001) afirma que a escolha desses recursos depende das necessidades e do comportamento dos clientes, e não apenas de estratégias que foram bemsucedidas do passado.

Novos recursos foram surgindo, de forma que os consumidores foram atingidos de diferentes maneiras, como o product placement, que, segundo Cowley e Barron (2008), apresenta vantagens sobre a propaganda tradicional na televisão. Além disso, o product placement tem atraído muitos investimentos das empresas, porque a propaganda é exibida durante o programa que os telespectadores estão assistindo. Dessa forma, inibe-se o efeito "zapping", que consiste na troca de canal incessante, como é possível fazer com comerciais tradicionais, durante os intervalos (PETROLL; PRADO, 2014). De forma geral, o objetivo do product placement é gerar associações positivas à marca e, em consequência, provocar atitudes positivas (COWLEY; BARRON, 2008).

Nas duas últimas décadas, é evidente o crescimento da audiência de séries de TV no mundo todo. Um exemplo disso é o surgimento de serviços de streaming de filmes e séries de TV, como a Netflix, que chegou ao Brasil em setembro de 2011 (G1, 2011). Estima-se que, em 2015, seu faturamento tenha ultrapassado em cerca de 250 milhões de reais o faturamento do Sistema Brasileiro de Televisão, o SBT (UOL, 2016).

De acordo com o Banco de Séries (2016), um dos maiores websites usados no Brasil para controle de calendário de séries, utilizado ativamente por mais de noventa mil usuários, há mais de onze mil títulos cadastrados e quase $60 \%$ desses títulos são americanos. O restante provém de cerca de quarenta países diferentes.

Brennan, Rosenberg III e Hementera (2004) afirmam, em sua replicação de estudo sobre product placement em filmes, que a cultura é um fator que provoca variações em pesquisas sobre o assunto, porque garante uma avaliação mais aprofundada. Em consideração a isto, é possível afirmar que as atitudes em relação ao product placement podem ou não ser similares em decorrência ao contexto em que é inserido, em virtude das diferenças culturais observadas.

Brasil e Estados Unidos apresentam culturas bastantes diferentes uma da outra. O Brasil é um país coletivista e que preza a convivência em grupos, tanto no ambiente pessoalquanto no profissional, enquanto os Estados Unidos têm características mais individualistas. Americanos valorizam a competição e o sucesso, e ser o melhor é o objetivo, enquanto brasileiros prezam mais a qualidade de vida e o pertencer a sociedade. Existem também diferenças em relação ao poder. No Brasil, a hierarquia é mais valorizada e as diferenças sociais são mais aceitas, enquanto nos Estados Unidos essa característica é menos comum (HOFSTEDE, 2017).

Em vista disso, este trabalho consiste em um estudo comparativo entre a percepção de brasileiros e americanos em relação ao uso do product placement. Para tal, analisaram-se o interesse despertado na marca, a intenção de compra, a percepção geral, a aceitação e a atitude de brasileiros e americanos em relação ao uso da estratégia de product placement em séries de TV. Por fim, foi estabelecido um comparativo entre a percepção dos dois grupos culturais estudados.

Embora esteja claro que a inserção de uso de produtos em filmes, séries e novelas na TV é uma das estratégias de marketing utilizadas hoje como meio de atingir o consumidor, o product placement ainda carece de aprofundamento teórico de suas aplicações. Através dos resultados obtidos, foi possível observar como os consumidores reagem à inserção de product placement em mídias televisivas. É também importante ressaltar que, a pesquisa, sendo realizada em dois países de culturas diferentes, proporciona resultados aos profissionais de marketing, de modo que possam atingir com mais assertividade em relação aos dois públicos através da estratégia do product placement.

No meio acadêmico, o trabalho tem relevância para estudos em novas áreas de marketing, onde cada vez mais é necessário se adequar aos novos hábitos adquiridos pelos consumidores e, assim, direcionar 
outras pesquisas para enriquecer o campo de estudo. Além disso, será possível identificar as reações dos usuários aos estímulos em relação ao uso da inserção de produto em séries de TV.

\section{Referencial Teórico}

\subsection{Product placement}

Nas últimas décadas, é tendência no marketing das empresas que as práticas de negócios sejam integradas, a fim de proporcionar uma comunicação que transmita a mesma mensagem aos clientes existentes e ao mercadoalvo. Essa integração é chamada de Comunicação Integrada de Marketing (CIM) e tem como aspecto importante a sinergia. Aplicar a CIM implica que todos os elementos de comunicação falem com uma única voz, ou seja, que não ocorra discrepâncias nas mensagens transmitidas por meios de comunicação diferentes (LUCK; MOFFATT, 2009). Além disso, é preciso definir qual seria a melhor abordagem e de que forma deve-se atingir o mercadoalvo (SHIMP, 2001). Uma dessas abordagens é o product placement.

Karrh (1998) define o placement como a inserção paga de marcas ou identificadores de marcas por meio de uso visual e/ou sonoro em programas de mídia de massa. Para D'Astous e Chatier (2000), product placement é a inclusão de produto, marca ou nome de uma empresa com intenções promocionais em filmes ou programas de TV. O uso do product placement está crescendo pela sua efetividade em atingir os consumidores e a audiência, estando presente em várias mídias, principalmente na televisão. O maior propósito dessa estratégia é fornecer reconhecimento ao produto/serviço de forma que a imagem fique "grudada" na cabeça da audiência. Depois de atingir com sucesso essa fase inicial, objetiva-se fazer com que o consumidor cultive pensamentos positivos sobre a marca (BABACAN; AKCALI; BAYTEKIN, 2012).

Davtyan e Cunningham (2016), em sua pesquisa, concluíram que os consumidores têm melhores atitudes em relação a product placements do que em relação a comerciais nos intervalos dos programas. Essa técnica tem se mostrado uma estratégia paralela para a entrada e fortalecimento das marcas na audiência de programas e shows, que crescem cada vez mais. É importante que o placement esteja em um contexto, para que não pareça absurdo, e, quando inserido na história, pode se tornar extremamente mais efetivo do que em propagandas na TV (PRICECONOMICS, 2013).

Muitas séries de TV de grande popularidade apresentam vários product placements. A primeira série original da Netflix, House of Cards, foi foco de uma matéria do Los Angeles Times, na qual é comparada a uma casa de product placement, em um trocadilho com o título da série (House of Placement), por seus inúmeros placements durante a sua primeira temporada (FLEISCHER, 2013; THE GUARDIAN, 2014).

\subsection{Percepção geral e aceitação do uso de PRODUCT PLACEMENT}

Gupta e Gould (1997), através de estudo realizado sobre utilização do product placement em filmes, relatam que a aceitação do uso da estratégia é influenciada pelo gênero, pelas atitudes e pelo número de filmes assistidos. Neste estudo, verificou-se que os homens e os telespectadores que assistiam aos filmes com mais frequência se mostravam mais aceitáveis aos placements. Os resultados apontaram, ainda, que os placements têm uma melhor performance que comerciais de televisão quando se fala sobre recall de marcas.

Russel (2002) ressalta que uma conexão maior do produto com os eventos que compõe a história ou parte dela, o plot da história (eventos que compõe a história), contribui de forma significante para a memória da marca. Quando a modalidade do placement combina com o plot, a inserção do produto/marca se torna mais natural e o consumidor faz menos esforço em analisar o porquê de estar ali. As categorias de placements também influenciam em como o consumidor percebe seu uso. Placements auditivos, com maior conexão de plot, e placements visuais, com menor conexão de plot, resultam em uma avaliação da marca mais favorável do que o uso contrário (RUSSEL, 2002; GILLESPIE; JOIREMAN, 2016).

Ham, Park e Park (2016) encontraram, em pesquisa com diferentes grupos de consumidores, que mulheres com menor nível de escolaridade têm tendências mais favoráveis ao product placement em geral. Porém, quando identificam o propósito comercial da inserção do produto, passam a apresentar uma percepção mais negativa. Ficando evidente, assim, a importância da conexão do placement como plot da série de TV. 
Em relação a diferenças culturais, Nelson e McLeod (2005) encontraram que pessoas de cor (hispânicos, asiáticos e negros) percebiam mais o uso de product placement e gostavam mais da estratégia do que caucasianos. Já Sung e Gregorio (2010) afirmam que afro-americanos com baixa renda tendem a ser mais positivos ao product placement.

\subsection{Atitudes em relação ao PRODUCT PLACEMENT}

Phelps e Hoy (1996) definem atitude como a predisposição para responder a uma determinada marca ou produto, positiva ou negativamente, depois de ser exposto a estímulos através da publicidade. A experiência prévia que o consumidor tem com a marca também influencia na atitude (FERREIRA; OLIVEIRA, 2012). Esse conceito aplicado ao product placement revela que quando os respondentes conhecem a estratégia têm tendência a ter atitudes favoráveis para com as marcas inseridas (KWON; JUNG, 2013)

Em complemento, Kwon e Jung (2013), afirmam, também, que quando existe uma socialização de consumidores, ou seja, quando a estratégia é comentada, é provável que os consumidores apresentem atitudes mais favoráveis à marca, o que reforça a memória da marca (PATTON, 2014). Segundo Gupta e Gould (1997), os respondentes que apresentaram uma melhor aceitação ao uso de product placement e na habilidade do filme transparecer uma ideia realista tendem a apresentar atitudes mais favoráveis aos produtos que foram apresentados no questionário do estudo.

A forma como os placements são inseridos nos programas também é um fator de influência, conforme os estudos de Russel (2002) e Gillespie e Joireman (2016), os quais defendem que o uso sutil e não intrusivo dos produtos gera atitudes mais positivas.

Nessa linha, Van Reijmerdsal et al. (2016) afirmam que existe uma diferença entre atitudes de adolescentes e adultos em relação ao uso de product placement quando a estratégia é divulgada ao público, ou seja, quando é sabido, previamente, que a marca está no programa. O estudo afirma que quando adultos estão cientes de que estão vendo uma estratégia publicitária, o seu senso crítico é elevado em relação à marca, levando a atitudes negativas também. Já adolescentes têm menor probabilidade de ativar seu senso crítico, por estarem mais envolvidos emocionalmente. Enquanto fica evidente que adultos tendem a criar uma visão mais crítica da marca inserida, adolescentes podem criar uma visão ainda mais fortalecida da marca quando há ciência da estratégia. Assim, Chan, Lowe e Petrovici (2016) sugerem que marketers considerem produzir vários tipos de placements, com variados níveis de proeminência, para alcançar vários segmentos e públicos.

\subsection{Interesse na marca e a intenção de compra através do PRODUCT PLACEMENT}

Machleit, Madden e Allen (1990) definem interesse na marca como o nível de interesse que o consumidor estabelece na marca e o nível de curiosidade que ele precisa para conhecer mais sobre ela. Diferentemente da atitude, o interesse não é uma avaliação cognitiva da marca, mas, sim, o que vem antes, facilitando o desenvolvimento das atitudes do consumidor.

Segundo Pham, Geuens e De Pelsmacker (2013), marketers devem, no geral, tentar aumentar o prazer emocional em suas estratégias de marketing, já que propagandas que despertam sentimentos mais agradáveis normalmente são as preferidas. Para os autores, os sentimentos despertados por propagandas interferem direta e indiretamente na avaliação das marcas e, na maior parte, estão ligados especialmente a mudanças de atitude em relação à marca.

Machleit, Allen e Madden (1993) relatam que o interesse despertado para marcas menos conhecidas influenciam as atitudes, salientando, porém, que existe uma ligação fraca entre efeitos despertados por propagandas com marcas consolidadas. Reforçam, ainda, que o interesse despertado pelas propagandas está mais associado ao interesse para com as marcas novas do que a marcas maduras. Por isso, estratégias para marcas mais familiares podem não funcionar da mesma maneira que para as menos conhecidas (DAVTYAN; CUNNINGHAM, 2016).

O objetivo principal de um anunciante do product placement é o aumento nas vendas do produto de uma determinada marca (BARNHARDT et al.; 2016), tendo por objetivo influenciar o público, além de ser 
uma ferramenta promocional de rápido crescimento em diferentes meios de comunicação. Além disso, o product placement se difere dos comerciais tradicionais por sua intenção persuasiva, que está mais explícita, aumentando os níveis de intenção de compra (CHAN; PETROVICI; LOWE, 2016).

Intenção de compra é a probabilidade de um consumidor comprar determinado produto ou marca (PHELPS; HOY, 1996; BELCH; BELCH, 2004). A intenção de compra varia de acordo com a experiência de cada consumidor, porém, segundo Schmitt e Zarantonello (2010), a atitude perante a marca é um fator importante para determiná-la. A atitude que se têm sobre a marca influencia positivamente, e de forma significativa, a intenção de compra (FERREIRA; OLIVEIRA, 2012).

Zhang (1996), em sua pesquisa, afirma que propagandas com mais humor tendem a despertar atitudes mais favoráveis à propaganda em si e à marca, por isso influenciam positivamente na intenção de compra. Dessa forma, product placements em sitcoms podem ajudar a melhorar as atitudes e aintenção de compra em relação à marca (DAVTYAN; CUNNINGHAM, 2016; CHAN; LOWE; PETROVICI, 2016).

Ham, Park e Park (2016) encontraram que um grupo de consumidores que apresenta maior memória do produto ou marca inserida apresentam baixa intenção comportamental sobre a marca, ou seja, a intenção de compra e de interesse não são relacionadas. Para atingir os consumidores de modo que apresentem maior intenção de compra, é necessário que primeiramente apresentem uma reação favorável à estratégia utilizada, ligando as atitudes em relação à propaganda e em relação à marca (BOCK et al., 2012).

\section{Método}

O método de pesquisa utilizado para o desenvolvimento do trabalho seguiu os pressupostos de Hair Jr. et al. (2009) e Malhotra, Birks e Wills (2012) no que tange aos estudos de natureza quantitativa, sendo caracterizada como uma pesquisa descritiva. Mais especificamente, um levantamento (survey) com corte transversal. A survey foi operacionalizada a partir de aplicação de questionário, que foi enviado eletronicamente, através de redes sociais, para o público respondente. O questionário foi construído e hospedado no site Google Drive, sendo, posteriormente, possível obter os resultados em um levantamento estatístico para tabulação dos dados.

O público respondente da pesquisa foi constituído por brasileiros e americanos que assistem séries de TV. A seleção dos respondentes foi feita através de amostragem por conveniência, de forma que o público foi selecionado por estar no lugar certo na hora certa, como em navegadores de internet (MALHOTRA; BIRKS; WILLS, 2012). Obteve-se um total de 290 questionários válidos, sendo 165 brasileiros e 125 americanos.

\subsection{Instrumento de Coleta}

O questionário, inicialmente, explicou o que é product placement. Depois, contou com questões de perfil, a fim de identificar algumas características dos respondentes, e questões gerais, para traçar o perfil de consumo de séries de TV. Para que o questionário fosse considerado válido, foi necessário definir algumas questões de checagem e filtro. A questão de checagem identificava se os respondentes compreenderam o significado de product placement. Nos casos de erro na pergunta de checagem, o questionário foi descartado. Após, como pergunta filtro, o respondente foi questionado se já percebeu o uso de algum placement durante algum episódio de série de TV. Em caso de resposta negativa, o questionário também foi descartado da amostra final.

Por fim, o instrumento de coleta abordou, em relação ao product placement, questões gerais e de aceitação (adaptados de GOULD; GUPTA; GRABNERKRATUER, 2000), de atitude (baseado em SAADEGHAZIRI; DEHDASHTI; ASKARABAD, 2013), o interesse despertado no consumidor (modificado de ZHAO; MHUELING; KAREKLAS, 2014; DODDS; MONROE; GREWAL, 1991) e a intenção de compra gerada pelo product placement (adaptado de ZHANG, 1996; BOCK et al., 2012).

O questionário, no que se refere aos construtos do tipo fechado, seguiu uma escala do tipo Likert de 5 pontos, em que cada respondente selecionou a opção que mais correspondia a sua percepção, variando entre concordo totalmente (5) e discordo totalmente (1) (MALHOTRA; BIRKS; WILLS, 2012). Por fim, em 
virtude de o questionário ser aplicado em dois países diferentes, foi escrito em português e em inglês. Para que não ocorressem divergências de interpretação, foi realizada a tradução reversa para os dois idiomas.

\subsection{Análise dos Dados}

Primeiramente, foi realizada a tabulação dos dados em planilha Excel para tratamento inicial e eliminação dos questionários não válidos, para depois serem tabulados no software SPSS, versão 22. Para que as amostras analisadas fossem homogêneas, realizou-se o cálculo de z-score para idade e quantidade de séries assistidas, a fim de identificar a existência de outliers que, segundo Hair Jr. et al. (2009), são os casos com escores muito diferentes em comparação ao restante dos casos. Os outliers foram eliminados para que os resultados finais das amostras fossem confiáveis.

Realizou-se uma análise descritiva dos dados a fim de apresentar o perfil dos respondentes, observar as suas características e, na sequência, seu perfil de consumo. Para tal, usou-se análises descritivas, como distribuição de frequência, média e desvio padrão, que consistem na obtenção de informações sobre a amostra (DOWNING; CLARK, 2010).

Posteriormente, as escalas foram analisadas para observar a percepção dos respondentes em relação aos construtos preestabelecidos durante o trabalho. Para verificação de confiabilidade de cada construto, realizou-se teste de confiabilidade para identificar a consistência interna das escalas, através do alfa de Cronbach. $\mathrm{O}$ alfa de Cronbach deve ser um número de 0 a 1 que indica o valor quando se calcula o valor de duas amostras aleatórias não correlacionadas (CRONBACH, 1951).

Para escalas usadas para comparação de dois grupos, valores do alfa encontrados entre 0,70 e 0,80 já são satisfatórios (BLAND; ALTMAN, 1997). Finalmente, para análise paramétrica dos construtos, realizou-se o teste $t$, que é um teste estatístico para comparação de médias entre dois grupos. Para análise dos resultados, considerou-se um nível de confiança de 95\% (RUXON, 2006).

\section{Apresentação dos Resultados}

\subsection{Perfil dos respondentes}

Primeiramente, realizou-se uma análise do perfil dos respondentes. Dos 276 respondentes, 70,3\% são do sexo feminino e $29,7 \%$ do sexo masculino. Em ambos países, a porcentagem de respondentes de mulheres é superior (69,9\% brasileiras e $70,8 \%$ americanas). A média de idade dos 276 respondentes é 22,06 anos e o teste t realizado não indicou diferença significante na média de idade entre os grupos $(t=1,273 ; p=0,204)$.

Os dois grupos foram avaliados em relação ao número de séries de TV e a quantos episódios assistem por semana. Conforme a Tabela 1, os brasileiros obtiveram maior média tanto ao número de séries de TV quanto ao número de episódios assistidos por semana.

Tabela 1 - Médias de brasileiros e americanos

\begin{tabular}{c|c|c}
\cline { 2 - 3 } \multicolumn{2}{c}{} & \multicolumn{2}{c}{ Brasileiros } & 7,57 \\
\hline $\mathbf{N}^{\circ}$ de séries de TV & 9,61 & 8,24 \\
\hline $\mathbf{N}^{\circ}$ de episódios & 10,20 & \multicolumn{2}{c}{ americanos } \\
\hline
\end{tabular}

Fonte: Dados da pesquisa (2017).

Através do teste $t$, identificou-se $t=1,969$ com $p=0,05$, indicando que há significância entre os grupos com relação ao número de séries de TV. São considerados significantes os valores de $p$ menores ou iguais a 0,05 (NANDHINI; SIVANANDAM, 2015). Já com relação ao número de episódios assistidos por semana, não existe diferença significante entre os grupos, já que $t=0,350$ e $p=0,727$.

Foram realizados, também, análises para comparar o perfil dos respondentes, analisando homens e mulheres de cada país. Em ambos os grupos, os respondentes do gênero masculino apresentaram média 
maior em relação à frequência com que identificam o uso de product placement em séries de TV, de 3,96 e 4,20 . Nos dois casos, houve diferença significante no resultado da análise de teste $t$, sendo $t=-2,134 ; p=0,034$ e t=-3,436; $p=0,001$, indicando que homens identificam o uso de product placement com mais frequência.

A Tabela 2 apresenta as médias separadas por gênero sobre o número de séries de TV e número de episódios assistidos. Os dados indicam que, com relação à quantidade de séries assistidas, dentro dos respondentes brasileiros, as mulheres apresentam média maior do que os homens. e o mesmo ocorre com a quantidade de episódios assistidos por semana.

Tabela 2 - Média de mulheres e homens entre os grupos

\begin{tabular}{l|c|c|c|c}
\cline { 2 - 5 } & \multicolumn{2}{c|}{ Brasil } & \multicolumn{2}{c}{ EUA } \\
\cline { 2 - 5 } & Mulheres & Homens & Mulheres & Homens \\
\hline Número de séries de TV & 10,43 & 7,72 & 7,55 & 7,63 \\
\hline Número de episódios & 11,28 & 7,68 & 10,39 & 8,48 \\
\hline
\end{tabular}

Fonte: dados da pesquisa (2017).

Em relação ao meio utilizado para o consumo de séries, é possível identificar que os serviços de streamings on-line e Netflix têm sido uma das escolhas da maioria dos respondentes (Figura 1). Para os americanos, a TV a cabo aparece como segunda opção, enquanto que, para os brasileiros, são os downloads.

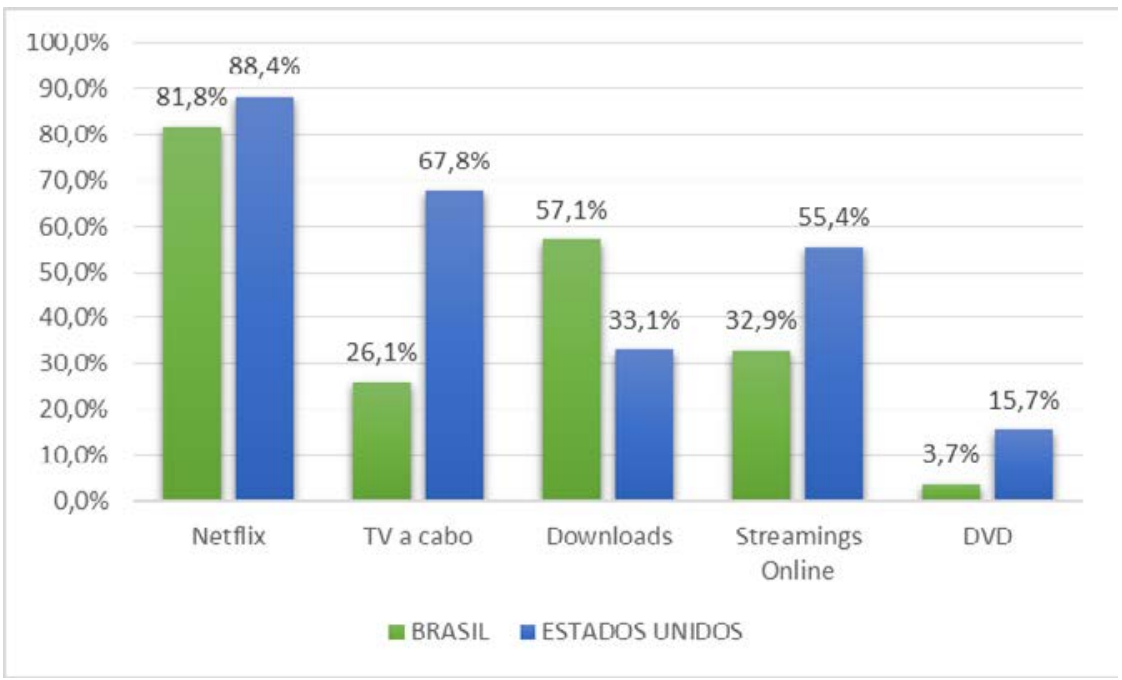

Figura 1 - Meio de assistir aos episódios

Fonte: Elaborado pelos autores (2017).

Por fim, para identificar o perfil, foi questionado se os respondentes conheciam o termo product placement, a fim de verificar o quanto estavam familiarizados com essa estratégia de marketing. Dos americanos, $84,2 \%$ conheciam previamente o termo, enquanto apenas $35,9 \%$ dos brasileiros conheciam. Acredita-se que isso se dá pelo termo ser de origem inglesa e não muito utilizado no Brasil.

Também foi questionada qual a frequência com que os respondentes identificavam o uso de tal estratégia em séries de TV, considerando-se 1 (quase nunca) e 5 (quase sempre). A média total encontrada foi de 3,71 no geral. A média de frequência dos brasileiros foi de 3,70, enquanto dos americanos foi de 3,76. $O$ teste $t$ não apontou diferença significante entre os grupos $(t=-0,501 ; p=0,617)$.

\subsection{Análise dos Construtos}

A Tabela 3 indica as médias obtidas pelos dois grupos culturais em relação aos cinco construtos analisados. 
Tabela 3 - Média dos construtos

\begin{tabular}{l|c|c}
\cline { 2 - 3 } & Brasileiros & americanos \\
\hline Percepção geral & 4,17 & 3,98 \\
\hline Aceitação & 4,01 & 3,87 \\
\hline Atitude & 2,65 & 3,24 \\
\hline Interesse despertado & 2,98 & 2,61 \\
\hline Intenção de compra & 2,65 & 2,35 \\
\hline
\end{tabular}

Fonte: Dados da pesquisa (2017).

O primeiro construto analisado se refere à avaliação geral que os respondentes têm em relação ao uso de product placement em série de TV, o qual foi medido por quatro afirmações. Antes da análise dos dados, verificou-se a consistência interna do construto através de teste de confiabilidade. $O$ alfa de Cronbach indicou resultado de 0,69 . Esse construto foi considerado pelo valor muito próximo do mínimo aceitável conforme a metodologia. Conforme a Tabela 3, os brasileiros apresentaram maior média e a análise paramétrica indicou diferença significante, já que $t=2,381$ com $p=0,018$, ou seja, a avaliação geral dos brasileiros em relação ao uso de product placement é significantemente mais positiva do que a dos americanos.

O segundo construto da pesquisa que avalia a aceitação dos grupos em relação ao uso de product placement em séries de TV contava com 5 afirmações. O construto indicou alfa de Cronbach de 0,70 quando realizado o teste para verificação da consistência interna. Assim como a avaliação geral, os brasileiros também obtiveram maior média em comparação aos americanos, indicando maior aceitação do uso do product placement em séries de TV. Após realização do teste t para esse construto, identificou-se que não existe diferença significativa entre os grupos em relação ao construto, já que $t=1,484$ com $p=0,139$.

Já a terceira questão a ser analisada, a atitude, era composta por quatro afirmações e obteve alfa Cronbach de 0,89 no teste de confiabilidade. Esse foi o único construto em que os americanos apresentaram maior média em comparação aos brasileiros, conforme mostra a Tabela 3. Com base nos dados, é possível afirmar que os brasileiros apresentam uma atitude mais negativa em relação ao uso de product placement, já que, além da média geral, as médias das quatro afirmações ficaram abaixo de 3 . A análise paramétrica indicou diferença significativa entre os grupos de respondentes, já que $t=-4,820$ com $p=0,000$.

O quarto construto mediu, através de cinco afirmações, o interesse despertado no consumidor pelo uso de product placement em séries de TV. Realizou-se teste para averiguação da consistência interna do construto, em que o alfa de Cronbach encontrado foi de 0,82. Os brasileiros obtiveram média mais alta em comparação aos americanos e, com relação a este grupo, é possível afirmar que uso de product placement provoca baixo interesse nas marcas, uma vez que as médias das cinco afirmações ficaram abaixo de 3. Em análise do teste $t$, encontrou-se diferença significante entre os grupos $(t=3,351 ; p=0,001)$, ou seja, o interesse despertado pela estratégia é maior para brasileiros.

Finalmente, o quinto construto, referente à intenção de compra do consumidor em relação ao uso de product placement em séries de TV, contava com 3 afirmações. O teste de confiabilidade também indicou consistência interna no construto, com alfa de Cronbach de 0,83 . Mais uma vez, a maior média foi do grupo brasileiro, e o teste $t$ também apresenta um resultado consistente com as médias, já que $t=2,433$ com $p=0,016$, indicando que existe diferença significante entre os grupos.

\section{Discussão de Resultados}

A partir da análise dos resultados, estabeleceram-se comparações entre os grupos investigados. O perfil dos respondentes encontrado neste estudo é consistente com pesquisas previamente realizadas, em que mulheres representam a maioria da amostra e que a média de idade é baixa, representando a faixa etária consumidora de séries de TV.

Na medida em que a maioria dos respondentes escolheu a Netflix como pelo menos um dos meios utilizados para assistir séries de TV (Brasil=81,8\% e EUA=88,4\%), há indícios de que existe correlação entre a Netflix ser a principal escolha dos respondentes eo aumento no uso de serviços de streamings pelo 
consumidor (FOLHA DE SÃO PAULO, 2017; VARIETY, 2015; TV AND MEDIA 2016). E devido ao fato de que, nos Estados Unidos, as emissoras de TV abertas disponibilizam gratuitamente episódios recentemente exibidos em sua programação normal, a TV a cabo e os streamings on-line possuem também uma grande representatividade, $67,8 \%$ e $55,4 \%$, respectivamente.

Já para os brasileiros que responderam à pesquisa, o download é o segundo maior meio usado pelos respondentes para assistir séries de TV $(57,1 \%)$ seguido dos streamings on-line $(32,9 \%)$. Apesar do crescimento de streamings legais nos últimos anos (PIFFERO, 2015), o download é um meio muito representativo do modo em que os brasileiros assistem os episódios das suas séries de TV (SAWADA, 2016; OLHAR DIGITAL, 2016; UOL, 2016).

Em relação à quantidade de séries assistidas, brasileiros apresentaram média significantemente maior do que os americanos. Em relação a episódios assistidos por semana, apesar dos respondentes brasileiros apresentarem média maior que os americanos nos dois casos, não foi encontrada diferença significante entre os grupos. Americanos, apesar de apresentarem médias menores na quantidade de séries e episódios assistidos, apresentaram média maior em relação à frequência com que identificam o uso do product placement.

Silveira (2012) sugere que, para entender o comportamento do consumidor em relação ao product placement, é necessário examinar como o comportamento de consumo dos consumidores é afetado pelos aspectos culturais. Nelson e Devanathan (2006) afirmam que audiências de diferentes países e culturas podem perceber o uso de product placement diferentemente, dependendo das experiências culturais. Identificou-se diferença significante entre quase todos os aspectos pesquisados comparando os dois grupos.

Na mesma linha, Samaha, Beck e Palmatier (2014) afirmam que relações de marketing também variam entre países e, segundo seu estudo, países em desenvolvimento, como o Brasil, apresentam resultados mais efetivos em comparação aos Estados Unidos e, por isso, marketers devem ajustar estratégias de acordo com seus mercados. Os resultados da pesquisa comprovam tal afirmação, uma vez que brasileiros perceberam melhor o uso da estratégia do que os americanos.

Em relação à aceitação do uso do product placement, não se encontrou diferença significante entre os grupos. As médias encontradas são consideravelmente altas, indicando boa aceitação da estratégia. Levando em consideração que as afirmações em relação ao construto de aceitação dizem respeito, principalmente, ao realismo que a utilização de placements propõe, é possível relacionar as médias altas de aceitação dos grupos à essa característica percebida.

Consumidores que conhecem a estratégia tendem a apresentar atitudes mais favoráveis ao product placement (GUPTA; GOULD, 1997; KWON; JUNG, 2013). Em concordância com isso, os americanos apresentaram atitudes significantemente mais favoráveis ao product placement, sendo que $84,2 \%$ deles afirmaram que já conheciam o termo previamente, enquanto apenas $35,9 \%$ dos brasileiros afirmaram que já tinham ouvido falar da estratégia. Americanos também identificam o uso de product placement com mais frequência do que brasileiros.

Nelson e Mcleod (2005) relacionaram o efeito que a propaganda exerce no consumidor diretamente à forma que ele avalia a propaganda em si. Dessa forma, é possível relacionar o resultado encontrado, em que brasileiros demonstram maior interesse no produto do que americanos, com a percepção geral sobre o uso de product placement, que é mais favorável do que a dos americanos.

Embora o consumidor apresente boas atitudes para como a marca, é necessário, também, verificar a percepção que tem da estratégia para que a intenção de compra seja maior (BOCK et al., 2012; GUPTA; GOULD, 1997). É possível, a partir disso, estabelecer uma relação entre a percepção e a aceitação da estratégia com a intenção de compra. Nos três casos, os brasileiros apresentaram médias maiores que os americanos, indicando que, como os brasileiros percebem melhor o product placement, também têm tendência a apresentar uma intenção de compra maior.

\section{Considerações Finais}

O uso da inserção de produtos e marcas em episódios de séries de TV (product placement) tem ganhado muito espaço, porque é um recurso a que o consumidor não tem como evitar ser exposto. O produto 
pode estar sendo usado/consumido pelo seu personagem favorito durante o episódio ou estar inserido no plano de fundo de uma cena. Quando existe coerência nessa colocação, o consumidor tem tendência a demonstrar uma boa percepção para com as marcas.

O propósito do trabalho foi identificar a percepção dos consumidores, brasileiros e americanos, em relação ao uso do product placement. De forma geral, identificou-se que existem diferenças significativas entre como os respondentes de cada país percebem e se comportam em relação ao uso da estratégia e também com relação às marcas que se utilizam dela. Brasileiros e americanos apresentam uma percepção positiva do uso do product placement em geral. Além disso, ambos apresentam uma boa aceitação ao seu uso. Quanto às atitudes em relação ao uso do product placement, americanos são mais favoráveis.

No que se refere ao interesse na marca, brasileiros tendem a apresentar um interesse maior. O mesmo ocorre em relação à intenção de compra. Porém, nesse último caso, as médias encontradas foram menores dentre todos os resultados. Nesse sentido, é possível que o uso do product placement não interferira de forma tão significativa na intenção de compra dos consumidores.

Os resultados deste estudo podem auxiliar marketers a entender melhor como utilizar o product placement. Brasileiros e americanos têm percepções diferentes ao seu uso, e, por isso, um uso generalista da estratégia pode não ser tão eficaz. As séries de TV, na sua maioria, são americanas, mas cada vez mais são consumidas pelo mercado internacional. Dessa forma, adequar o modo como se utiliza a inserção das marcas, visando também outros países alcançados pelo conteúdo, pode ser uma estratégia interessante para empresas que estão presentes também no mercado mundial.

É importante que marketers entendam como aplicar o recurso da melhor forma. Séries que exigem maior atenção combinadas com um placement muito proeminente, por exemplo, podem causar irritação no telespectador ao invés de reações positivas. Além disso, o telespectador aprecia a noção de realidade que o product placement proporciona às histórias, por isso é importante que o profissional adéque o seu produto de forma que o consumidor entenda como natural a sua presença em cena.

Este trabalho apresentou diversas limitações no decorrer do seu desenvolvimento. Encontrou-se que existem diferenças significantes entre a percepção de brasileiros e americanos sobre o product placement, porém podem existir muitos outros fatores que influenciam nessa diferença além dos citados na discussão de resultados. Além disso, o uso de uma única imagem para a ilustração do conceito de product placement no questionário pode ter influenciado os respondentes, já que se tratava de um placement proeminente. Dessa forma, dada a possibilidade de que o respondente pode ter considerado a imagem um padrão, é interessante que pesquisas futuras consideram outras formas de placement.

Além disso, pela amostra ser heterogênea e de maioria feminina, os resultados de comparação entre gêneros não são os ideais para que se generalizem as conclusões. Da mesma forma, deve-se reconsiderar a baixa média de idade dos participantes. Diante disso, sugere-se que novas pesquisas sejam realizadas, a fim de comparar grupos com distribuição mais homogênea de gênero e de diferentes faixas etárias.

\section{Referências}

AMARAL, S. A.. Marketing da informação: entre a promoção e a comunicação integrada de marketing. Inf. \& Soc.:Est., João Pessoa,v. 18, n. 1, p.31-44, jan./abr.2008.

BABACAN, E.; AKCALI, S.; BAYTEKIN, E. Product placement as a rising marketing Communication Activity: $\mathrm{n}$ Assessment on Television Serials. Procedia - Social And Behavioral Sciences, [s. I.], v. 62, p.1319-1331, Oct. 2012.

BANCO DE SÉRIES (Comp.). Modern Family - 6x16: connection Lost. Disponível em: http:// bancodeseries.com.br/index. php?action=se\&serieid=2981\&episode=136\&pag =top\&type;=. Acesso em: 19 out. 2016.

BARNHARDT, T.M. et al. The effects of product placement in fictitious literature on consumer purchase intention. Psychology \& Marketing, [s. I.], v. 33, n. 11, p. 883-898, Nov. 2016.

$\mathrm{BELCH}, \mathrm{G}$.; BELCH, M. Advertising and promotion: an integrated marketing communications perspective. $6^{a}$ ed.. New York: The McGraw-Hill Companies, 2004. 
BLAND, M.; ALTMAN, D. Statistics Notes: Cronbach's Alpha: Cronbach's Alpha. British Medical Journal, [s. I.], v. 314, n. 7080, p. 572-573, Feb.1997.

BOCK, G. et. al. The progression of online trust in the multi-channel retailer contextand the role of product uncertainty. Decision Support Systems, [s. I.], v. 53, n. 1, p. 97-107, Apr. 2012.

BRENNAN, S.; ROSENBERGER III, P.; HEMENTERA, V. Product placements in movies: an Australian consumer perspective on their ethicality an acceptability. Marketing Bulletin, [s. I.], v. 15, n. 1, p.1-16, 2004.

CHAN, F.; PETROVICI, D.; LOWE, B. Antecedents of product placement effectiveness across cultures. International Marketing Review, [s. I.],v. 33, n. 1, p.5-24, 2016.

COWLEY, E.; BARROW, C. When product placement goes wrong: the effects of program liking and placement prominence. Journal of Advertising, [s. I.], v. 37, n. 1, p.89-98, 2008.

CRONBACH, L. Coefficient alpha and the internal structure of tests. Psychometrika, [s. I.], v. 16, n. 3, p. 297-334, Sep. 1951.

D'ASTOUS, A.; CHARTIER, F. A study of factors affecting consumer evaluations and memory of product placements in movies. Journal of Current Issues \& Research In Advertising, [s. I.], v. 22, n. 2, p.3140, 2000.

DAVTYAN, D.; CUNNINGHAM, I. An investigation of brand placement effects on brand attitudes and purchase intentions: Brand placements versus TV commercials. Journal of Business Research, [s. I.], v. 70, p.160-167, Jan. 2017.

DOODS, W.; MONROE, K.; GREWAL, D. The effects of price, brand, and store information on buyers' product evaluation. Journal of Marketing Research, [s. I.], v. 28, n.3, p. 307-319, Aug. 1991.

DOWNING, D.; CLARK, J. Estatística aplicada. 3ª ed. São Paulo: Saraiva, 2010.

FERREIRA, P.; OLIVEIRA, R. A influência da experiência de marca na atitude e intenção de compra: o caso Red Bull. Book of Proceedings - TMS Algarve, Algarve, v. 1, p.303-315, 2012.

FLEISCHER, M. 'House of Cards,' or more like house of product placement? 2013. Disponível em: http://articles.latimes.com/2013/may/03/entertainment/la-et-st-house-of cardsnetflixproductplacement-20130503. Acesso em: 19 out. 2016.

BRENTANO, L. Netflix chega ao Brasil por R\$ 15 ao mês. São Paulo:G1,2011 Disponível em: http:// g1.globo.com/tecnologia/noticia/2011/09/netflix-chega-ao-brasil-por-r-15por-mes.html. Acesso em: 22 set. 2016.

GOULD, S.; GUPTA, P.; GRABNER-KRÄUTER, S. Product placements in povies: a cross-cultural analysis of Austrian, French and American cnsumers' attitudes toward this emerging, international promotional medium. Journal of Advertising, [s. I.], v. 29, n. 4, p.41-58, Winter, 2000.

GILLESPIE, B.; JOIREMAN, J.; MUEHLING, D. The moderating effect of ego depletion on viewer brand recognition and brand attitudes following exposure to subtle versus blatant product placements in television programs. Journal of Advertising, [s. I.], v. 41, n. 2, p.55-65, Mar.2012.

THE GUARDIAN. As seen on TV: why product placement is bigger than ever.2014. Disponível em: https://www.theguardian.com/tvandradio/2014/jun/24/breaking-bad-tv-product-placement. Acesso em: 19 out. 2016.

GUPTA, P.; GOULD, S. Recall of products placed as prizes versus commercials in game shows. Journal of Current Issues And Research In Advertising, [s. I.], v. 29, n. 1, p.43-53, May 2007.

HAIR, J. F. et. al.. Análise multivariada dedados. 6. ed. Porto Alegre: Bookman, 2009.

HAM, C-D.; PARK, J. S ; PARK, S. How U.S. Consumers respond to product placement: cluster analysis based on cognitive and attitudinal responses to advertising in general. Journalism \& Mass 
Communication Quarterly, [s. I.], v. 29, n. 1, p.1-29, Sep. 2016.

HOFSTEDE, G. Brazil in comparision with United States. 2017. Disponível em: https://geert-hofstede. com/brazil.html. Acesso em: 07 jun. 2017.

KARRH, J.. Brand placement: a review. Journal of Current Issues and Research in Advertising, [s. I.], v. 20, n. 2, p.31-49, 1998.

KWON, E.; JUNG, J-H. Product placement in tv shows: the effect of consumer socialization agents on product placement attitude and purchase intention. Online Jornal on Communication and Media Technologies. [s. I.], v. 3, n. 4, p. 88-106. Oct. 2013. Disponível em: https://www.ojcmt.net/download/ product-placement-in-tv-shows-the-effect-of-consumer-socialization-agents-on-product-placement. pdf. Acesso em: 26 out. 2016.

LUCK, E.; MOFFATT, J. IMC: Has anything really changed? A new perspective on an old definition. Journal of Marketing Communications, [s. I.], v. 15, n. 5, p. 311-325, Nov. 2009.

MACHLEIT, K. A. ; MADDEN, T. J.; ALLEN, C. T.. Measuring and modeling brand interest as an alternative aad effect with Familiar Brands. Advances in Consumer Research, [s. I.], v. 17, p.223-230, 1990.

MACHLEIT, K.; MADDEN, T.; ALLEN, C. The mature brand and brand interest: an alternative consequence of Ad-Evoked affect. Journal of Marketing, [s. I.], v. 57, p.72-82, Oct. 1993.

MALHOTRA, N. K.; BIRKS, D.; WILLS, P. Marketing research: applied approach. 4. ed. New York: Pearson, 2012.

NANDHINI, M; SIVANANDAM, S. N. An improved predictive association rule based classifier using gain ratio and T-test for health care datadiagnosis. Sadhana, [s. I.], v. 40, n. 6, p.1683-1699, Sep. 2015.

NELSON, M.; MCLEOD, L. Adolescent brand consciousness and product placements: awareness, liking and perceived effects on self and others. International Journal of Consumer Studies, [s. I.], v. 29, n.6, p. 515-528, Nov. 2005.

OLHAR DIGITAL. $\mathbf{8 0 \%}$ dos internautas brasileiros fazem download de filmes e séries na internet. 2016. Disponível em: https://olhardigital.uol.com.br/noticia/80-dos-internautas-brasileiros-fazemdownload-de-filmes-e-series-na-internet/60865. Acessoem: 18 maio 2017.

PATTON, D. A Study of the effect of product placement in television shows and its influence on consumer behavior. 2014. Bachelor of Arts. Faculty of the Communications Studies Department California Polytechnic State University, San Luis Obispo, 2014.

PETROLL, M.; PRADO, P. Um ensaio teórico sobre placement televisivo e seus efeitos sobre o consumidor. RAC - Revista de Administração Contemporânea, Rio de Janeiro,v. 18, n. 2, p.176-195, Mar./Abr. 2014.

PHAM, M.; GEUENS, M.; PELSMACKER, P. The influence of ad-evoked feelings on brand evaluations: Empirical generalizations from consumer responses to more than 1000 TV commercials. International Journal of Research in Marketing, [s. I.], v.30, n. 4, p.383-394, Dec. 2013.

PHELPS, J.; HOY, M. The Aad-Ab-PI relationship in children: the impact of brand familiarity and measurement timing. Psychology \& Marketing, [s. I.], v. 13, n. 1, p. 77-101, Jan. 1996.

PÍFFERO, L. Disputa entre serviços de TV por streaming promete mais conteúdo original e variedade de programas: mercado brasileiro ainda é pequeno, mas cresce rapidamente.2015. Disponível em: https://gauchazh.clicrbs.com.br/cultura-e-lazer/noticia/2015/02/disputa-entre-servicos-detv-por-streaming-promete-mais-conteudo-original-e-variedade-de-programas-4708066.html. Acesso em: 27 maio 2017.

PRICEONOMICS. The Economics of Product Placements. MGM Studios; BMW, 2013. Disponível em: https://priceonomics.com/the-economics-of-product-placements/. Acesso em: 19 out. 2016. 
RUSSELL, C. Investigating the effectiveness of product placement in television shows: the role of modality and plot connection congruence on brand memory and attitude. Journal of Consumer Research, [s. I.], v. 29, n. 3, p. 306-318, Dec. 2002.

RUXTON, G. D. The unequal variance t-test is an underused alternative to Student's t-test and the MannWhitney U test. Behavioral Ecology, [s. I.], v. 17, n. 4, p.688-690, July/Aug. 2006.

SAADEGHVAZIRI, F.; DEHDASHTI, Z.; ASKARABAD, M. R. K. Web advertising. Journal of Economic And Administrative Sciences, [s. I.], v. 29, n. 2, p. 99-112, 2013.

SAMAHA, S. A.; BECK, J. T.; PALMATIER, R. W.. The role of culture in international relationship marketing. Journal of Marketing,[s. I.], v. 78, n.5, p.78-98, Sep. 2014.

SAWADA, T. Avanço do streaming sinaliza fim da pirataria. 2016. Disponível em: < http://link.estadao. com.br/noticias/cultura-digital,avanco-do-streamingsinaliza-fim-da-pirataria,10000069428>. Acesso em: 18 maio 2017.

SILVEIRA, R. Product placement in movies: a cross cultural study between Brazil and the usa. 2012. . Dissertação (Mestrado em Administração de Empresas) - Fundação Getúlio Vargas, São Paulo, 2012.

SUNG, Y.; GREGORIO, F. New brand worlds: college student consumer attitudes toward brand placement in films, television shows, songs, and video games. Journal of Promotion Management, [s. I.], v. 14, n.1-2, p. 85-101, 2008.

SCHMITT, B.; ZARANTONELLO, L. Using the brand experience scale to profile consumers and predict consumer behavior. Journal of Brand Management, [s. I.], v. 17, n. 7, p. 532-540, Mar. 2010.

SHIMP, T. Comunicação integrada de marketing. In: CZINKOTA, M. R. et al. Marketing: as melhores práticas.Porto Alegre: Bookman, 2001.CD-ROM.

THE MEDIA BRIEFING. How America watches tv: 8 trends to be aware of. 2015. Disponível em: https://www.themediabriefing.com/article/how-america-watches-tv. Acessoem: 17 nov. 2016.

THE NEW YORK TIMES (Ed.). How many scripted tv shows in 2015? A precise number, and a record. 2015. Disponível em:

http://www.nytimes.com/2015/12/17/business/media/how-many-scripted-tv-showsin-2015-a-precisenumber-and-a-record.html?_r=1. Acesso em: 18 out. 2016.

UOL Netflix fatura R \$1,1 bi no Brasil e ultrapassa o SBT. 2016. Disponível em: http://tvefamosos.uol. com.br/noticias/ooops/2016/01/11/netflix-fatura-r-11-binobrasil-e-ultrapassa-o-sbt.htm. Acesso em: 22 set. 2016.

VAN REIJMERSDAL, E. A. et al. This is advertising! Effects of disclosing television brand placement on adolescents. Journal of Youth and Adolescence,[s. I.], v. 46, n. 2, p. 328-342, Feb. 2017.

VARIETY. Streaming overtakes live tv among consumer viewing preferences: study.2015. Disponível em:

http://variety.com/2015/digital/news/streaming-overtakes-live-tv amongconsumerviewing-preferencesstudy-1201477318/. Acesso em: 28 nov. 2016.

ZHANG, Y. Responses to humorous advertising: the moderating effect of need for cognition. The Journal of Advertising, [s. I.], v. 25, n. 1, p.15-32, Sping 1996.

ZHAO, G.; MUEHLING, D.; KAREKLAS, I. Remembering the good old days: the moderating role of consumer affective state on the effectiveness of nostalgic advertising. Journal of Advertising, [s. I.],v. 43, n. 3, p.244-255, Aug. 2014. 